\title{
HACIA UNA METÓDICA DE CARÁCTER PROBLÉMICO EN LA CLASE DE EDUCACIÓN FÍSICA ${ }^{1}$
}

\author{
TOWARDS ONE METHOD OF PROBLEM NATURE IN PHYSICAL EDUCATION CLASS
}

\author{
Miguel Ángel Molano², Mauricio Robayo ${ }^{3}$ \\ Diana Feliciano ${ }^{4}$, Guillermo Camacho $^{5}$ \\ Grupo de Investigación COMPLEXUS
}

Resumen

El presente escrito parte de una revisión de diversos enfoques problémicos, hacia el fundamento de una metódica de carácter problémico en la clase de Educación Física, retomando como fuentes de problematización: el movimiento en sí, la interacción con el compañero(s), la comprensión de las situaciones de juego o de instrucciones, y la actitud personal frente a las situaciones planteadas en clase. Con estas se estructura un conjunto de estrategias para situaciones problémicas, que son desarrolladas en clase. De su aplicación se desprenden unas moralejas a tener en cuenta.

Palabras claves: educación física, métodos, estrategias metodológicas, situación problémica.

Abstract

The written present start from a review of diverse problem approaches, towards foundation one method of problem nature Physical Education class, taking again as sources of problem: movement itself, interaction with partners, comprehension of game situations or instructions, and the personal attitude in class situations. With these sources we structure a set of strategies for problem situations that are developed in class. With his application emerge a few morals to bearing in mind.

Keywords: physical education, methods, methodological strategies, problem situation.

Fecha de recepción: 6 de Septiembre de 2010

Fecha de aprobación: 4 de Noviembre de 2010

“Todo problema trae consigo un don. Afronto problemas, porque necesito sus dones"

Richard Bach (Ilusiones)

${ }^{1}$ Proyecto de investigación DGP-CIUP, FEF - 052-07.

${ }^{2}$ Licenciado en Educación Física, Especialista en Pedagogía y Didáctica de la Educación Física, Especialista en Docencia Universitaria, Profesor de la Universidad Pedagógica Nacional, desde 1994. mimolab2000@hotmail.com

${ }^{3}$ Licenciado en Educación Física, Especialista en Pedagogía y Didáctica de la Educación Física, Magíster en Sociología de la Educación. Profesor de la Universidad Pedagógica Nacional.

${ }^{4}$ Licenciada en Educación Física, Especialista en Pedagogía y Didáctica de la Educación Física, Magíster en Currículo y evaluación. Profesora de la Universidad Pedagógica Nacional, desde 2007.

${ }^{5}$ Licenciado en Educación Física, Especialista en Desarrollo Humano. Profesional - Asistente de investigación grupo complexus. 


\section{Antecedentes de pedagogía problémica}

Generalmente se toma como ideal del conocimiento, al conocimiento científico, y por ende, podríamos decir que los enfoques problémicos en la enseñanza, se plantean la tarea de reconstruir didácticamente "el método" por el cual se da el conocimiento y sus posibilidades de aprendizaje por el ser humano. La "problematicidad" del "método" se ha tomado como el garante de un conocimiento, desde el método propio de las ciencias denominadas empírico-analíticas, en un marcado énfasis cognitivista, tomando distancia de los problemas cotidianos en que se forma el estudiante.

En las diferentes teorías del aprendizaje, así como en los diferentes enfoques epistemológicos, el carácter problémico del conocimiento (y su aprendizaje) se ha abordado de diferente manera. Así pues, los problemas se pueden resolver por simple ensayo y error, nos dicen los asociacionistas, o por comprensión súbita, nos dice la gestalt o la teoría de la información nos plantea la resolución de problemas por medio de estrategias algorítmicas ${ }^{6}$ y heurísticas ${ }^{7}$ previamente aprendidas que dan lugar a una nueva posibilidad, provocando un nuevo aprendizaje que servirá en otras situaciones.

Majmutov, plantea, desde una perspectiva materialista-dialéctica, que la contradicción es la fuerza motriz de la enseñanza problémica, siempre y cuando una contradicción se presente a la conciencia del estudiante como tal, y tome conciencia de esta como una dificultad a resolver.

La contradicción en el pensamiento se presenta siempre no en forma de tarea, sino como un problema; la contradicción se entiende como una dificultad subjetiva sólo en la situación problémica que puede ser provocada no solamente por tareas cognoscitivas o prácticas de cualquier tipo. Por consiguiente, junto a la contradicción externa señalada

${ }^{6}$ 1. Identificación de variables del problema. 2. Seguimiento de pasos predeterminados ¿fases del método científico? 3. Búsqueda de recursos necesarios para dar solución al problema.

${ }^{7} 1$. Conjeturas o supuestos básicos. 2. Ensayo y error, o ensayo mental de las opciones. 3. Empleo de analogías, simulaciones, etc. Integrando estos procesos algorítmicos y heurísticos, se selecciona la estrategia se aplica, se evalúan los resultados y se retroalimenta el proceso. Esto, desde el paralelo con el computador. Teoría cibernética. por M.A. Danilov debe encontrarse la interna, que incluya la condición antes indicada, es decir, que sea la fuente del desarrollo de las fuerzas cognoscitivas del alumno y la fuerza motriz del aprendizaje (Majmutov, 1984, p. 59).

Esta perspectiva nos hace entender que las situaciones problémicas emergen en un campo de fuerzas en tensión. En el caso de la enseñanza, entre las exigencias que desde la tarea se hacen al estudiante, llevándolo a un estado de desequilibrio, frente a las condiciones cognitivas del estudiante, que le llevan a implicarse en la situación, y poner en juego sus recursos para resolverla.

Desde las teorías cognitivas del aprendizaje, Hans Aebli nos plantea la resolución de problemas, diciendo que el ser humano, en relación con su saber, siempre tiene "lagunas", puntos en blanco no relacionados con el conjunto del saber, que no presentan una hilación coherente con las redes significativas en torno a un fenómeno. Retomando el principio gestáltico de cierre, nos dice Aebli que siempre buscamos cerrar estos espacios en blanco, vacíos o lagunas y es allí donde surge un estado problémico que se puede orientar (Aebli, 2001, p. 240).

Lo anterior nos lleva a comprender que son más (siempre muchos más) los vacíos en esta red, que los tejidos significativos. El arte de enseñar apuntará a hacer ver (retomando el principio gestalt de alternancia) estos puntos vacíos, a interesar al otro, tanto como uno, por descubrir el trasfondo de esas lagunas desconocidas, e indagar por sus relaciones con lo conocido, en un proceso abductivo ${ }^{8}$.

Según plantea Vigotsky (Tudge, En Moll, 1993, p. 177) nos enfrentamos a lo desconocido desde nuestra zona de seguridad. Por "acción de la cultura", (lengua, norma, instituciones) somos "jalonados" a una zona de desarrollo proximal, que nos permite, por "nuestra acción", pasar a una zona de desarrollo potencial, que será nuestra nueva zona de seguridad.

${ }^{8}$ Desde quien interpreta, el análisis y la síntesis son insuficientes. El cerebro, de por sí, parece operar en un sentido abductivo. A partir de indicios, conjeturas, relaciones incompletas entre el todo y las partes, genera hipótesis progresivas, en la búsqueda de dar sentido a lo percibido. Y se va afinando en la búsqueda de indicios. Conjeturar es constituir diversos posibles sentidos (Pierce, El animal simbólico). 
cíproco, en la cual se dinamiza el desarrollo entre los procesos filogenéticos, (acerbo primario del sujeto) y los procesos ontogenéticos (acerbo secundario). Este planteamiento de Vigotsky se complementa con Piaget, cuando este explica como, en tanto un proceso de maduración se de, a partir de una situación de desequilibrio cognitivo, se generan descompensaciones que obligan al sujeto a asimilar y acomodarse, a partir de su acción, dándose una acomodación por reelaboración de esquemas de pensamiento (Piaget, 1998). Pero, cabe observar que este estado de desequilibrio cognitivo, sólo se genera cuando su acerbo, sus propias condiciones son insuficientes para enfrentar las condiciones y exigencias del medio, es decir, se enfrenta a un problema. Aquí se hace evidente la necesidad y conveniencia de plantear la enseñanza desde un enfoque problémico.

En las interacciones entre el sujeto y el objeto, se da un proceso dialéctico entre la búsqueda de la estabilidad por el sujeto, que, (lo evidenciamos todos los días) se resiste al desequilibrio desde todas las formas y argumentos posibles, frente a las fuerzas sociales y materiales del entorno que desde sus exigencias propician desequilibrios en el sujeto. Pero a la vez, las inquietudes, las dudas y tensiones internas del sujeto, buscan transformar un entorno demasiado estable y que no responde a sus intereses y/o necesidades.

Vemos que la metodología problémica se ha fundamentado desde las diferentes teorías del aprendizaje, cada cual dándole un matiz diferente, un carácter distinto, y desde su perspectiva, prescribe y lee diferentes procesos en la metodología problémica. Pero es común a todas ellas la intención de reconstruir los diferentes procesos científicos retomando así la metodología de formación en el pensamiento científico. Subyacen allí, pensamos, diferentes concepciones de lo que sería hacer ciencia y del científico. Desde la educación física, un carácter problémico de la enseñanza, no apuntaría a "la formación de un científico", sino al desarrollo de una mentalidad reflexiva sobre el cuerpo, sobre las condiciones y posibilidades personales, sobre la relación con el otro, pero además, no desde la teoría (aunque también) sino desde la experiencia.

Por lo anterior, consideramos que desde la educación física no es suficiente la acción del estudiante en un proceso "ordenado" en el aprendizaje de una técnica biomecánicamente (científicamente) prescrita, en un proceso escalonado, por una vía formal y progresiva del paso uno, paso dos, sino que hay que mirar la acción, no como acto aislado, generado por el sujeto-estudiante, sino como una unidad dinámica entre el estudiante y su entorno, mediado por una tarea de movimiento, en la cual se teje una situación donde el estudiante pone en juego su acerbo y condiciones frente a las condiciones y exigencias planteadas por la situación. Nos dice Néstor Bravo:

[...] una pedagogía problémica postula la conexión entre investigación y enseñanza en la dialéctica concreta, cuya lógica real de la producción del conocimiento puede ser conocida y apropiada a partir de la determinación de la contradicción dialéctica, en tanto expresión de las múltiples y diversas fuerzas y tendencias que explican el desarrollo del conocimiento y la cultura como algo no acabado, definitivo y totalmente coherente (Bravo, 1997, p. 16).

Se plantea la necesidad de "desarrollar el pensamiento y la comprensión de la realidad en la dinámica de sus contradicciones reales" (Bravo, 1997, p. 17). Así pues, un enfoque problémico de la enseñanza-aprendizaje supone la producción y recreación del saber como proceso crítico constructivo, donde la lógica del proceso de enseñanza va más allá de los pre-determinados "pasos del método", hacia el estudio y la comprensión de los conflictos, los núcleos de tensión en que se genera el conocimiento en su propia dinámica de relaciones, interacciones, de continuidades y discontinuidades, convergencias-divergencias, diálogos-discusiones, cuestionamientosacuerdos, normas-criterios, reflexiones-acciones, reflexiones sobre las acciones, en fin, sobre aquellos núcleos de relaciones que propicien la problematización.

Lo anterior nos lleva a reconocer en nuestro estudiante su carácter "trágico". Tragedia en el sentido dado en la Grecia clásica, era la condición del ser humano enfrentado a dos verdades igualmente válidas y, situado en la contradicción, verse obligado a elegir, asumiendo el "a costa de que", que lo fuerza a valorar el acto de la decisión 9 .

Pues sólo entendiéndose el hombre como un ser trágico es que se permite a sí mismo acceder a la duda, a la construcción de certezas, llegando a los

\footnotetext{
${ }^{9}$ En forma exacta, este planteamiento se puede ver en Zuleta, E. Arte y filosofía.
} 
cambios profundos en su propio ser. Es en la dinámica de las contradicciones, donde se generan los cambios conscientes (Molano, 1991, pp. 35-36).

A este ser trágico lo complementa la comprensión de que el hombre es un ser "dialógico". Un ser eminentemente cultural que no se basta a sí mismo para comprender el mundo y necesita del "otro", de "los otros". Bien nos lo recuerda Paulo Freire, cuando dice: "ya nadie educa a nadie, así como tampoco nadie se educa a sí mismo. Los hombres se educan en comunión, y el mundo es el mediador" (Freire, 1983, p. 86). No es un sujeto previamente dado, totalmente estructurado quien conoce, sino un sujeto en desarrollo, en cambio permanente, tanto en su estructura, como en sus formas de organización e interacción con su entorno ecológico y social y que no reproduce textualmente y pasivamente el saber cultural, configurándose así en un campo de fuerzas físicas, bióticas, sociales, y subjetivas en las que se enmarca su desarrollo.

\section{Aproximaciones a la idea de problema}

En la revisión sobre los enfoques problémicos en la enseñanza, identificamos dos enfoques clásicos según el enfoque epistemológico que subyace.

Una primera que se explica: el problema está en la realidad objetiva. En este marco, los problemas son previos al sujeto, quien tiene que emplear los instrumentos que le da el entorno para resolverlo. La segunda, que se explica que los problemas están en el sujeto que se los plantea, en tanto que se encuentre en desequilibrio, generado por la incertidumbre, la necesidad, o el deseo.

Habría un tercer enfoque, inter-estructurante, que plantea que los problemas surgen o derivan de la interacción entre el sujeto y el mundo. Esto quiere decir que el problema surge en el marco de unas relaciones que se estructuran entre el sujeto y su mundo (biológico, físico y social).

La inter-estructuración, desde nuestra perspectiva, más que un método, es un enfoque epistemológico de la enseñanza, que deriva, por supuesto en una metódica (conjunto de métodos, estrategias y/o principios que guardan relación entre sí, desde una concepción de la enseñanza, del aprendizaje, del conocimiento) que reconoce una dialéctica entre pro- cesos hetero y auto-estructurantes, presentes en la dialógica de la enseñanza-aprendizaje, teniendo en común procesos de interdependencia, corresponsabilidad, problemicidad, que en su dialógica devela una dinámica de conflictos. El conflicto surge de la problematización que se desarrolle sobre la contradicción, dado que el estudiante no se hace necesariamente consciente de la contradicción y no se desequilibra. Nos dice Muska Mosston:

Si el clima cultural en el que se educa el alumno erigió barreras que obstruyen su capacidad de respuesta, un problema potencialmente relevante no necesariamente crea en el alumno la excitación que espera el maestro. Ha de existir una relevancia emocional (Mosston, 1982, p. 12).

Este hecho nos reitera que la contradicción sólo se supera en la dinámica de la acción: acción de la cultura-acción del estudiante (figura1).

Un "yo actúo" sobre el mundo, sobre el entorno (entorno físico-biótico-social) que no es, no puede ser independiente de las retroacciones que el entorno ejerce sobre su propia acción, donde su intención, entra en juego con las condiciones de posibilidad de su puesta en ejercicio, frente a otras intenciones, su voluntad frente a otras voluntades, en bucles dialógicos que dinamizan esta relación en situaciones de conflicto y/o consenso.

Pero a su vez, el objeto (el ambiente) no es pasivo frente al sujeto. Como campo de fuerzas sociales, bióticas, físicas, retro-actúa sobre el sujeto posibilitando condiciones de desequilibrio que por su propia acción, el sujeto tendrá que recuperar, generando, a su vez procesos de recursión que le garanticen una cierta estabilidad y en los cuales, propicia nuevas condiciones potenciales para enfrentar a su entorno. 


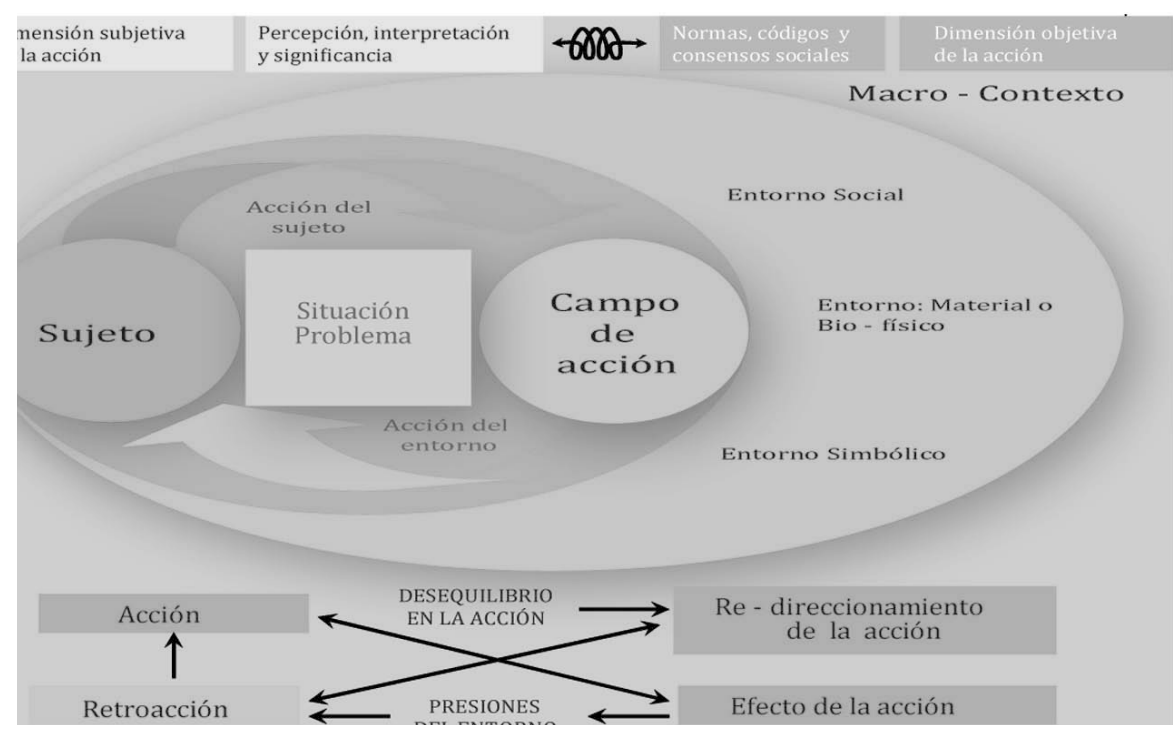

Figura 1. La acción: unidad dinámica del sujeto con su entorno.

Generalmente tomamos como problema a una situación que se nos presenta inconclusa, o aquello que no encaja en un estado de cosas, o lo que se presenta a nuestro pensamiento como incomprensible, o tomamos como problema aquello que es superior a nuestras fuerzas, o a nuestros argumentos.

Seguramente todas las situaciones anteriores son problemáticas, pero entrar a definir el concepto de problema, nos exige mirar su fundamento, sus características, y el sentido. Esto nos lleva a mirar el problema en el marco de una relación humana con el mundo, es decir, que un problema es, ante todo una emergencia en la relación sujeto-mundo. Una situación que se teje en esta interacción, con un carácter de insuficiencia del acerbo propio, o del medio, de insuficiencia en la comprensión, o en la incompletud que dificulta la significación; donde en últimas, las partes: sujeto-objeto, yo mundo, individuo-el otro, no encajan. Situación que nos enfrenta a una contradicción. Así pues, el conflicto es inherente a la relación, pero nos hacemos impermeables a el. No lo vemos. Lo que hace el planteamiento problémico de la situación de enseñanza, es hacer explícita, visible la contradicción (figura 2).

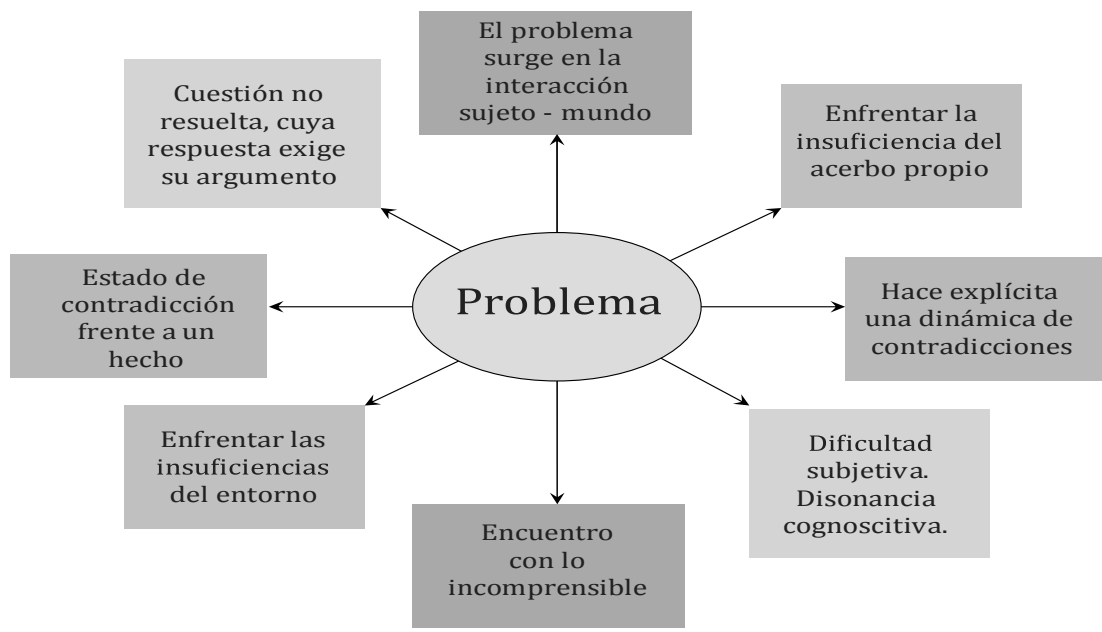

Figura 2. Diferentes interpretaciones de qué es un problema.
Un problema implica manejo de procedimientos, aptitud en las interacciones, estado de incertidumbre frente a la situación, en la relación dudacerteza, y exige la búsqueda de la coherencia interna en la resolución del problema.

Se entiende aquí que un problema es una situación de desequilibrio del sujeto frente a las condiciones del entorno, sea por carencias en sí mismo frente a las exigencias del medio, o por carencias del medio frente a las exigencias-necesidades del sujeto, que le lleva a crear en sí o recrear en el medio, condiciones para un nuevo equilibrio (figura 3 ).

Esta situación de desequilibrio, se entiende como un problema siempre en relación. Es decir, no surge en el sujeto, de manera aislada, ni tampoco es un hecho previamente dado por el entorno. Es en la dinámica de la acción (acción de la cultura-acción del sujeto o en nuestro marco: acción del maestro-acción del estudiante) donde se conforma el problema como un "tejido situacional" que se concreta en una "tarea". Es allí donde el maestro, le da a la tarea un carácter problémico, en el marco de una situación de clase, haciendo explícita una contradicción a superar, desde una intención pedagógica orientada a la formación de algún proceso de desarrollo. 


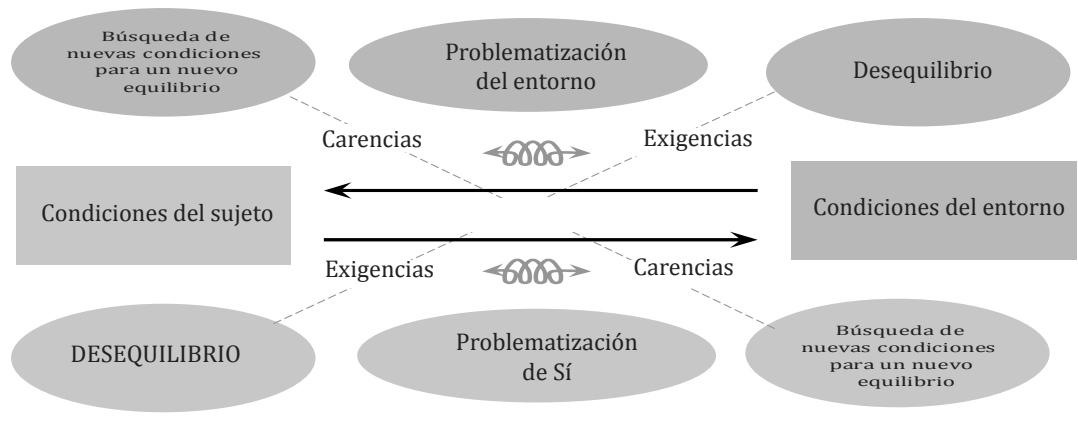

Figura 3. El problema como desequilibrio del sujeto frente al entorno.

La tarea se entiende como una actividad, componente particular de un sistema de saber, seleccionado de manera intencionada por el maestro como mediación entre un propósito educativo y los procesos de desarrollo del estudiante. En la educación física, se toma por tarea en un sentido genérico, desde el grueso de la educación física, hasta el juego o ejercicio más elemental. La tarea responde a una intencionalidad pedagógica, por ende no es un fin en sí misma. Es un dispositivo de intervención sobre las relaciones sociales de la escuela.

La tarea se caracteriza por ser:

- Estructurada: exige-reta las condiciones in situ del estudiante, desde sus características estructurales.

- Flexible: ajustable a sus condiciones de aplicación.

- Dinámica: en tanto permite su desestructuración y reestructuración.

- Procesual: conlleva en sí misma un proceso y es parte de un proceso.

Pero la tarea, si bien como estructura básica ha de contemplar los aspectos procedimentales, planteada desde un enfoque problémico, más que una actividad, es una situación en torno a lo inconcluso, incompleto, a la carencia, que hace explícito un conflicto. Dado que un aprendizaje, en su origen, no es independiente de la situación (significativa) en que se da, la situación problémica planteada, como un conjunto de relaciones entre sujetos y significados, acciones, retroacciones, hechos, no se planteará al estudiante de manera totalmente estructurada, favoreciendo la divergencia, aún en el cumplimiento de las condicionantes de la situación problémica planteada. En este marco, una pregunta, por ejemplo, será una mediación entre el estudiante y la tarea.

En este sentido, la tarea planteada como una situación problémica se caracteriza por:

- Propiciar el diálogo y la crítica.

- Propiciar el trabajo en equipo, sin asfixiar el aporte individual.

- Retro-actuar, con preguntas orientadoras, sobre los estudiantes, reorientando sus formas de relación, constreñimientos a la acción con un sentido de exigencia a lo superior.
- Un pensamiento divergente, o mejor, en tensiones entre convergencia y divergencia. Un marcado énfasis en la convergencia polariza, no permite integrar y llegar a una tercera opción.

\section{Configuración de una metódica de carácter problémico en la clase de Educación Física escolar}

En el marco de una "metódica" se articulan un conjunto de estrategias metodológicas en coherencia con una concepción de la enseñanza, que está, a su vez, articulada a una concepción epistemológica.

Así pues, desde una epistemología inter-estructurante, el conocimiento se estructura en el sujeto, en la dinámica presente entre las exigencias del medio al sujeto que lo desequilibran y conflictúan, y las acciones del sujeto conducentes a recuperar su equilibrio.

Cabe recordar que la enseñanza "es un proceso internamente contradictorio" (Majmutov, 1984, p. 33), entre la eterna tendencia de impartir el conocimiento como pre-dado, estático, que busca transmitir conclusiones ya preparadas, frente a la incertidumbre, a un saber inconcluso, que reta la imaginación y le exige adecuarse a lo nuevo.

Partimos de que una situación problémica, se genera a partir de unas "fuentes de problematización". Estas se entienden como los tejidos situacionales que en la clase son propicios para hacer explícitos unos núcleos de tensión propios del desarrollo. En el caso de la educación física, consideramos aquí diferentes fuentes de problematización: el "movimiento" en sí, la "interacción" con el compañeros(s), la "comprensión" de las situaciones de juego o de ins- 
trucciones, y la "actitud personal" frente a las situaciones planteadas en clase (figura 4).

1. En un primer nivel, consideramos el "movimiento en sí", partiendo de la base de que en este se expresan las limitaciones propias y los constreñimientos que impone la tarea al movimiento del estudiante, desde las reglas de juego, las condiciones de ejecución técnica de un gesto, los diferentes niveles de estructuración de la tarea, la variabilidad de las condiciones de ejecución, así como las posibilidades de transferencia de una acción a otras condiciones o situaciones. De alguna manera, el movimiento en sí mismo se hace problema, pero no enseñamos al estudiante a ser procesual y metódico, a entenderse en el proceso, a aprender de sus errores, a pensar. De esta fuente de problematización, nos planteamos una primera estrategia metodológica: la problematización del movimiento. En esta estrategia metodológica, buscamos privilegiar el movimiento en sí, y dos núcleos de tensión propios del movimiento: la maduración 12 aprendizaje, y las condiciones iniciales 60 condiciones potenciales ${ }^{10}$.

2. En un segundo nivel consideramos: los problemas emergentes en la interacción con los otros, posibilitando situaciones de cooperación-solidaridad a oposición-competencia, que entran a hacer más ricas y complejas las situaciones, además de poner en juego la posibilidad de acuerdos-desacuerdos, convergencias-divergencias, diálogo-discusión.

El sujeto que actúa (en interacción con otros) tiene que enfrentarse a otros centros de significación y de decisión. Todo cambia dado que la incertidumbre procedente de las conductas de los demás, y la influencia del medio físico inerte y determinista no son de la misma clase. Los procesos de decodificación semiotriz difieren considerablemente en ambos casos, pues los intercambios significativos, las fintas, las metacomunicaciones, las relaciones paradójicas sólo pueden manifestarse en situacio-

Esta complejidad situacional de la interacción con el otro, es potencialmente rica en fuentes de problematización, originada en la incertidumbre sobre la acción del otro, sea compañero o adversario, o como posibilidad de confrontación de las posibi-

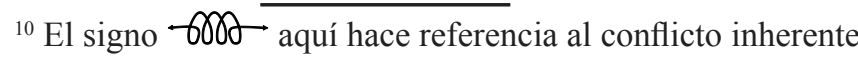
a una relación dialéctica. Conflicto que se expresa en relación de fuerzas a la vez "antagonistas" y "complementarias".

lidades propias con las del compañero. De esta fuente de problematización, nos planteamos una segunda estrategia metodológica: la problematización de la interacción. En esta estrategia metodológica, buscamos privilegiar las situaciones que permiten problematizar las diferentes formas de relación, al interior del grupo, a partir de dos núcleos de tensión propias de la interacción: la Individualidad @or socialización, y el interés particular 100 interés general.

3. En un tercer nivel, estaría la comprensión. En la educación física se presenta ambigüedad en la lectura del movimiento en sí. Un gesto, una situación considerada válida en un deporte, en otro es considerada una falta, o los fundamentos teóricos explicados, difieren frente a sus condiciones de aplicación. En una situación de juego, el niño tiene que considerar muchas variables, que va comprendiendo en la medida en que se desarrolla el juego, pero que muchas veces exigen una mentalidad táctica formada (malicia indígena) para reaccionar adecuada y rápidamente en esa situación concreta.

La "tarea" como problema tiene una carga simbólica tanto por parte del sujeto y su grupo, como por el maestro. Desde esta carga simbólica, el estudiante se aproxima a la tarea para aprehenderla, y en su "proceso de manipulación” y de asimilación de las características de la tarea "objeto de conocimiento", empieza a acomodar sus "esquemas de acción"11 aprehendiendo conocimientos, desarrollando aptitudes y valoraciones que a su vez re-elaboran esas "cargas simbólicas", generando representaciones construidas por el mismo, en el marco de la clase.

Es así como en las interacciones entre estudiantemaestro-tarea, en la clase se entreteje un universo simbólico propio, significativo para sus participantes, desde el cual se aproximan a la comprensión de la tarea, que se va haciendo más compleja en el proceso.

Este entramado simbólico que se genera en la clase no es ajeno al conflicto, al contrario: en él se generan unos núcleos de tensión: el maestro tiene un acerbo en su lenguaje, que le permite manejar los diferentes elementos, conceptos y categorías propios de la complejidad de la tarea a enseñar, frente al lenguaje

\footnotetext{
${ }^{11}$ Esquema de acción, en la teoría Piagetiana, son "trazas sensorio-motoras, repertorios parciales de acción ya estructurados en el sujeto, que son transferibles a nuevas situaciones, en nuevos procesos de reorganización de la acción" (Aebli, 1995, p.162).
} 
cotidiano que el estudiante maneja, y en la distancia entre esos dos lenguajes se teje el universo simbólico de la clase, generando tensiones en la explicación ford comprensión. Así mismo, tensiones en los procesos de retroacción Wor autorregulación. De esta fuente de problematización, nos planteamos una tercera estrategia metodológica: la problematización de la comprensión. En esta estrategia metodológica, buscamos privilegiar las situaciones de desequilibrio e incertidumbre frente a los procesos de aprendizaje, a partir de dos núcleos de tensión propias de la comprensión: la duda certeza y los códigos del maestro -00 códigos del estudiante.

4. En otro nivel ubicamos los "problemas existenciales". En la clase de Educación Física se presentan permanentemente situaciones donde está en juego un aspecto personal. Confrontar el cuerpo propio con los otros, "es que yo soy tímido", el "no puedo" que se expresan ante una posición invertida, la renuncia a salir de sus zona de seguridad, limitada a sus dominios cotidianos, el reconocer en los otros las carencias propias así como las potencialidades, son todas estas, fuentes de problematización, en las que el conflicto emerge o se hace explícito en la problematización que se desarrolla sobre la contradicción. El estudiante no es necesariamente consciente de la contradicción. Es en la situación problema donde se le hace patente, el conflicto que le suscita. Estas situaciones, mal manejadas, dejan frustraciones imborrables, pero, en un proceso reflexivo por el profesor, así como en un proceso consciente por el estudiante, propician una riqueza inmensa de situaciones formativas. De los problemas existenciales, como fuente de problematización, nos planteamos una cuarta estrategia metodológica: la problematización del sentido. En esta estrategia metodológica, buscamos privilegiar las situaciones que posibiliten la reflexión sobre el sentido de la experiencia, a partir de dos núcleos de tensión propios del sentido de la existencia: la adaptación al medio - 00 adaptación del medio y el interés y las expectativas 1000 exigencias y condiciones del medio.

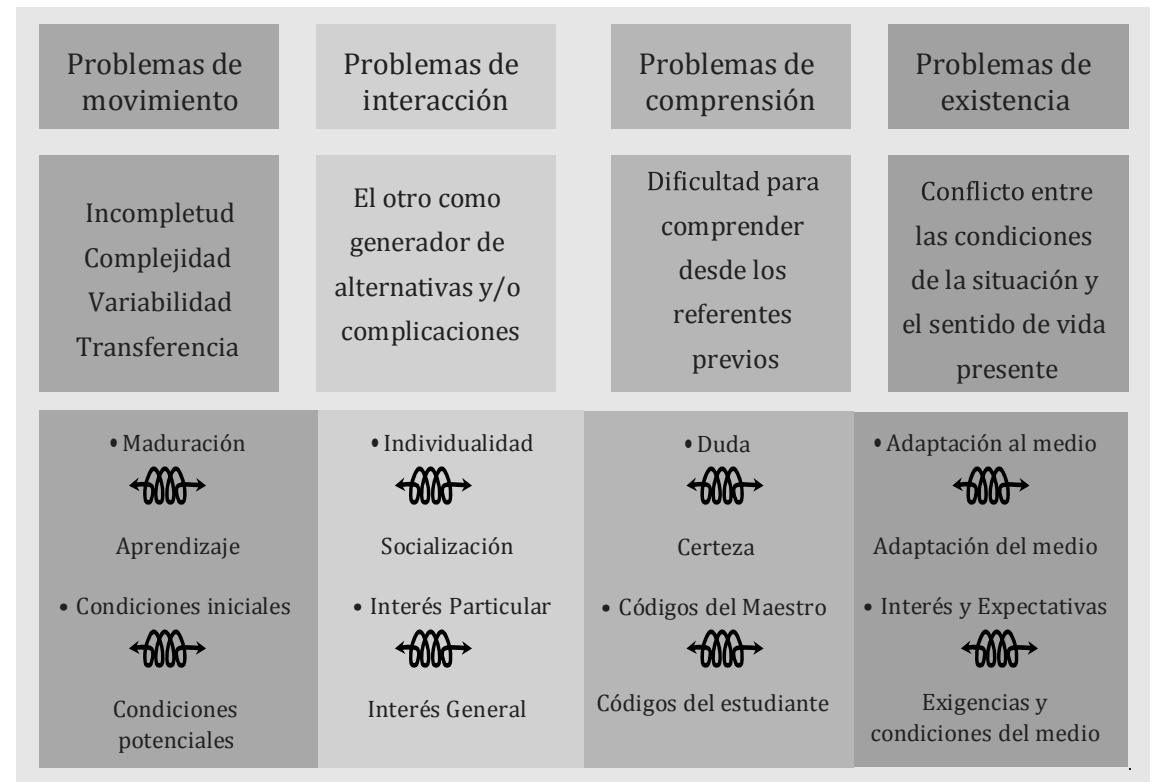

Figura 4. Fuentes de problematización y sus núcleos centrales de tensión.

\section{Los núcleos de tensión frente a las fuentes de problematización}

¿Cómo abordar el complejo de la socialización en el ser humano? Este proceso de socialización entre el cuerpo y la cultura, mediado por las relaciones de poder (regla, norma) en tensión con los procesos de empoderamiento (educación, formación), entre el código lingüístico y las formas expresivas, (lenguaje-expresión) entre el afecto-desafecto (emocionalidad: pulsión-represión) que redunda en arraigos-desarraigos, implicación-rechazo con el entorno cultural. Una interpretación problémica de este conjunto de relaciones y mediaciones, nos remite a la identificación de los núcleos de tensión que emergen al interior de estas relaciones.

$\mathrm{Al}$ revisar las teorías del desarrollo, se identifican tres fuerzas que orientan el desarrollo: las fuerzas biológicas, las fuerzas sociales, y las fuerzas que emergen de la subjetividad que se construye. Así pues, en la educación física, el cuerpo se construye en el cruce de caminos entre la subjetividad, los imperativos biológicos, la cultura y su extensión espacio-temporal-objetual, cuyas relaciones están mediadas por el tejido simbólico propio de la cultura. En este cruce de caminos, en la clase se ponen en juego unos núcleos de tensión propios del desarrollo, que pueden ser fuentes de problematización en el planteamiento de las tareas propias de la clase. Limitaciones fisiológicas, limitaciones en el campo de la conciencia, que dificultan la representación del movimiento, limitaciones en la apropiación del tiempo, el espacio y los objetos (figura 5). 
Hacia una metódica de carácter problémico en la clase de Educación Física

Miguel Ángel Molano / Mauricio Robayo / Diana Feliciano /Guillermo Camacho

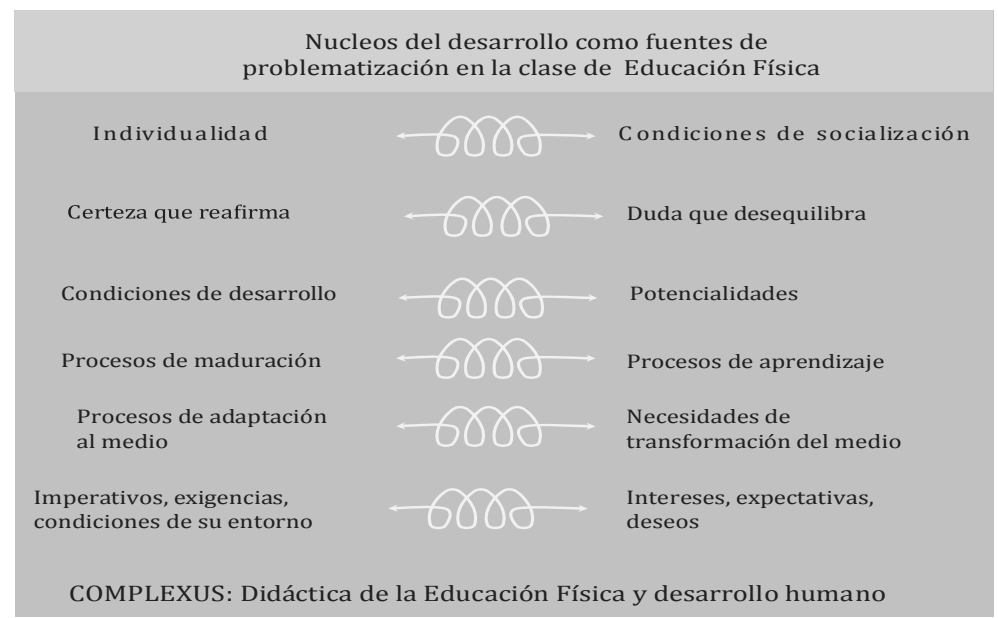

Figura 5. Núcleos de tensión en el desarrollo como fuentes de problematización.

Mirando la figura anterior, se identifican un conjunto de núcleos del desarrollo, que expresan las tensiones propias de las fuerzas que actúan en nuestro estudiante, con nuestro estudiante. Es decir, que él toma parte activa de ese conflicto, y en un estadio de su desarrollo, primará una fuerza frente a la otra, pero estas fuerzas lo colocan permanentemente en desequilibrio.

\section{Supuestos que subyacen en un método problémico}

- Desde una perspectiva del desarrollo humano no totalmente predeterminada, donde la linealidad y ascendencia de las fases de los desarrollos es jalonada por un campus de tensiones... desde unas interacciones sociales que no excluyen el conflicto, la duda, el temor, la divergencia.

- Una concepción del conocimiento como una construcción no totalmente terminada... y que permite soluciones alternativas, posibilitando la interdependencia entre la solución y las condiciones del estudiante(s) (Mosston, 1982, p. 211). Esto lleva a la conclusión de que todas las respuestas pueden ser correctas, si enmarcan en las condiciones planteadas para la situación. El conocimiento entendido no como reproducción de un saber previamente dado, sino como producción, o mejor, co-producción en comunidad (Freire, 1983, p. 86).

- El aprendizaje, si bien es un proceso cognitivo individual, está determinado por las situaciones sociales que le condicionan en sus procesos y en sus contenidos. (Median algunos aprendizajes previos, como saber trabajar en equipo, saber escuchar, comprender el mismo aprendizaje como un proceso que permite que el sujeto pueda aprehenderlo). En sus contenidos, está determinado por el universo simbólico del grupo social en el cual se origina ese aprendizaje (el estudiante hace una "media luna" en clase de gimnasia, y en la práctica del Capoeira, esta misma destreza tiene otro sentido y técnica, otra estética, que contradice el ideal bio-mecánico de esta destreza en la gimnasia).

\section{Principios de aplicación}

El planteamiento de la tarea como situación problémica, implica partir de las características del desarrollo del potencial humano propias del grupo.

- La E.F. no se puede quedar en el plano del acondicionamiento físico, sino que debe suponer la construcción de un tejido simbólico desde el cual el estudiante llegue a una comprensión lo más completa posible del movimiento, y por el movimiento, de otros procesos de su desarrollo del potencial humano. Revalorando la empiria, (como experiencia), como carácter fundamental de la educación física, que no se puede subsumir a un proceso reflexivo, pero si ha de propiciarlo y potenciarlo.

- No existe, generalmente, un modelo explicativo "único", para la resolución de los problemas. La fortaleza de la argumentación, la permanente reflexión sobre los vacíos conceptuales y de explicación, y la contrastación con las otras posibles explicaciones, ha de llevar a la progresiva apropiación del saber. Se han de privilegiar los procesos comunicativos en el grupo, así como un ambiente de confianza mutua en las capacidades entre sí, al interior del grupo.

- En la dialógica estudiante-maestro, cada uno ha de preguntarse a sí mismo, y en la dialógica, aportar su respuesta (maestro que no se interroga está muerto como maestro, estudiante que no interroga su saber, está muerto como estudiante). Si el estudiante no se pregunta, no puede preguntar al maestro y entonces el maestro pregunta. Lo propio en un modelo es quien aporta la pegunta.

- No dar la solución (problematizar, inducir a la pregunta, a la duda en el saber previo). Esperar las primeras 
exploraciones del estudiante y retomarlas. Resaltar las condiciones de en que se ha planteado el problema, dado que muchos estudiantes tienden a saltarse alguna regla condicionante de la situación. Considerar el número de soluciones válidas que tenga el problema.

- Planteamiento de las metas a conseguir. Planteamiento del problema y su nivel de dificultad. Información necesaria para encarar la búsqueda. Condiciones, normas y límites en los que se van a desenvolver el problema. La técnica de enseñanza será por indagación.

- Tener en cuenta el afán de superación. Considerar el agente provocador de la curiosidad. Tener en cuenta el reto. Exposición del tema-problema y sus puntos de interés. Presentar situaciones nuevas (curiosidad). Proporcionar parte de la información. Plantear una actividad como reto (conflicto). Limitar las posibilidades en la ejecución. Plantear hipótesis. Qué ocurriría si...

- La construcción de un planteamiento didáctico para conseguir la disonancia cognitiva-ruptura de la zona de de seguridad-desequilibrio en el estudiante, exige que frente a la tarea el maestro plantee preguntas, llevando gradualmente a que sea el estudiante quien se plantee las preguntas. Esto, conducente a que sea el estudiante quien se las plantee ante nuevas situaciones (Mosston, 1982, p. 165).

- La noción de "esquemas de acción", como referentes previos al aprendizaje, exige que los estudiantes tengan unas condiciones previas que les permitan afrontar el problema con éxito.
Es evidente que no inventamos todo de la nada, más bien componemos nuestras secuencias de acción a partir de elementos que hemos almacenado en nuestro saber sobre acciones o nuestra memoria de ellas. A estos los denominamos esquemas de acción. Son, por decirlo así, elementos de acción prefabricados. [...] transferibles a nuevas condiciones (Aebli, 2001, p. 162).

En el análisis estructural de la tarea, planteada como situación problémica, es necesario plantear unos referentes previos, sean de carácter procedimental, sean de carácter conceptual, que permitan asimilar la complejidad del problema planteado.

\section{Estructuración de la materia}

El planteamiento de situaciones problémicas en la clase de Educación Física, exige pensarla en términos de proceso, a un mediano plazo, donde los contenidos vayan ganando en sentido. Aunque excluye muchos contenidos posibles para la clase, consideramos el esquema de la figura 6 como un mínimo común de acuerdo.

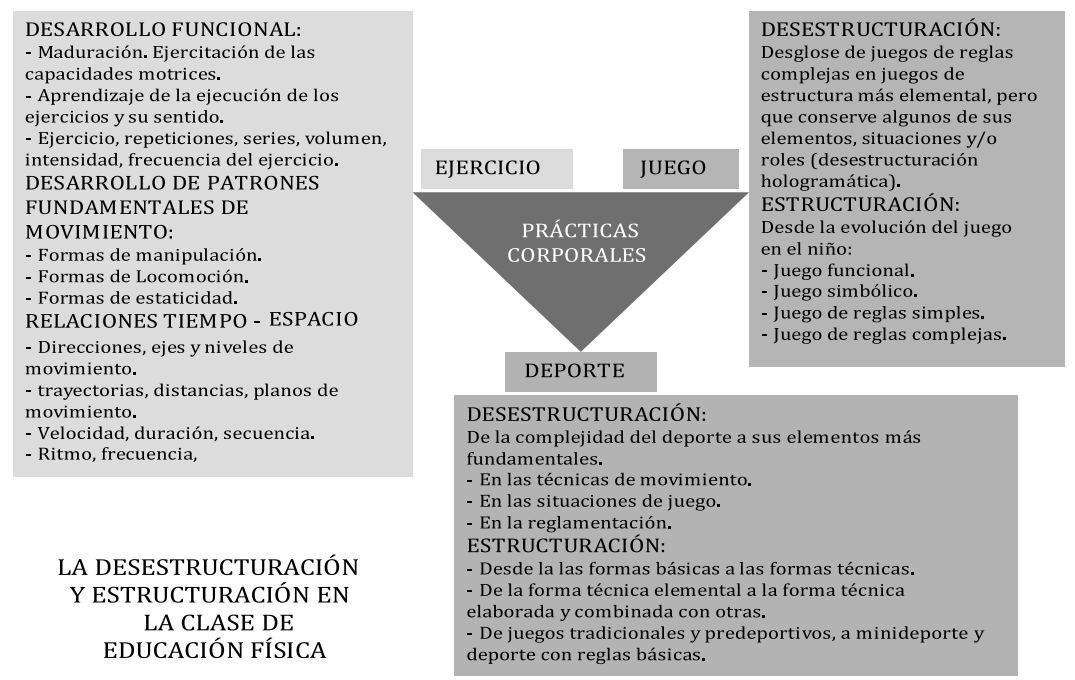

Figura 6. Desestructuración-estructuración de los contenidos en educación física.

Así pues, se estructurará teniendo en cuenta:

Niveles, progresivamente, en la búsqueda de formar en unos dominios determinados para un planteamiento problémico. Se está diciendo aquí que un planteamiento problémico de situaciones de clase, exige determinados procesos previos, como el trabajo en equipo, un clima de confianza en el saber del maestro por los estudiantes, confianza de ellos entre sí, y de alguna manera, en sí mismos. La cultura del consumo acostumbra dar todo masticado, y los estudiantes esperan eso, indefectiblemente. Cuando el maestro no responde a esta expectativa, hay resistencias, desconfianza en su saber, (diarios de campo) y apatía. Esto conlleva entonces a plantear un proceso gradual. 
Determinando fases pertinentes y niveles de dominio con respecto al problema: nivel 1, co-implicación; nivel 2, co-determinación; nivel 3, co-construcción.

a. En la primera fase "co-implicación", se busca un tanteo exploratorio de la situación, sus exigencias, condiciones, reconocimiento de las condiciones propias para enfrentar la situación problema, búsqueda de alianzas y acuerdos en el grupo para su aproximación (fase emocional).

b. En la segunda fase "co-determinación", se busca un acercamiento más analítico, en la búsqueda de los elementos claves para la resolución del problema. Se asignan roles y tareas, acordes con la complejidad del problema.

c. En la tercera fase "co-construcción", se eligen y confrontan las diferentes opciones de resolución, se practica, y se buscan aplicaciones a otras situaciones (transferencia).

Se entienden estas fases como no secuenciales, sino como momentos del proceso en que se privilegian determinadas acciones; donde el grupo, desde sus características de desarrollo, genera posibilidades distintas de acción. Con los más chicos, prima la primera fase, con un carácter más exploratorio, en los primeros grados del bachillerato prima la segunda fase, y con los más grandes, la tercera. Así pues, se entienden como momentos por los que pasa cada taller, en los cuales se privilegian determinados procesos, sin excluir los anteriores, o limitar el paso a los siguientes. Esto es así, por la diversidad del grupo, sus formas particulares de organización para el trabajo, sus demoras en conciliar intereses, que hacen que las situaciones de convergencia y/o divergencia sean disímiles.

Fases de la problematización

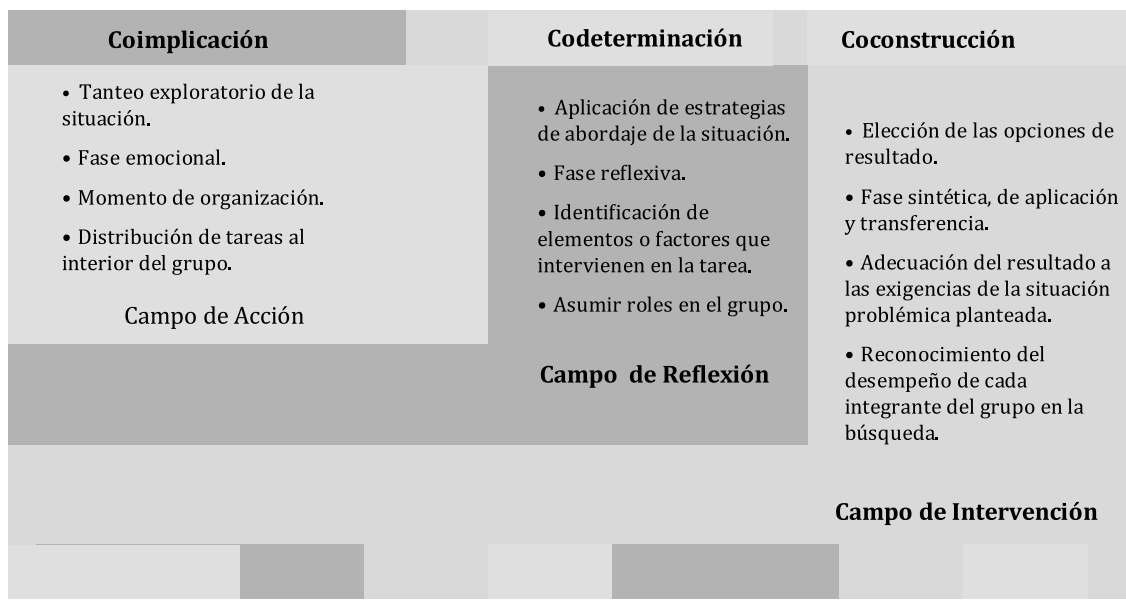

Figura 7. Las fases de la problematización

\section{En progresiones}

- Al principio una serie de problemas motrices aislados y coyunturales. De allí, pasamos a problematizar situaciones de clase, en el marco de la relación con los otros, en el marco de la comprensión de la situación de juego (por ejemplo). Posteriormente pasaría- mos a problematizar situaciones más profundas, en el marco de la existencia (problemas existenciales).

- Según la edad: infantil y primer ciclo, al no existir modelos de ejecución es mejor enseñar mediante la búsqueda y la exploración. Es pertinente plantear niveles de solución del problema.

- El planteamiento problémico de las tareas de clase, coexiste con formas directivas, co-directivas y exploratorias por el estudiante, en su proceso de aprendizaje. Es claro que enfocar problémicamente las situaciones de clase, exige del maestro estar dispuesto a estructurar las tareas dejando campo abierto a la emergencia de situaciones propicias a la problematización. Lo que lleva a una intermitencia en la aplicación del método problémico.

- Nivel de dificultad del problema: adecuar según nivel de los estudiantes, para que pueda generarse un nivel de problematización por el estudiante. Desarrollar progresivamente, buscando que la solución del problema no sea conocida de antemano por los estudiantes. Que no sea demasiado fácil, pero tampoco imposible de solucionar. Que las condiciones de evaluación sean coherentes con los procesos trabajados.

\section{Procedimientos claves}

- Definir un problema central. Determinar las categorías de análisis que intervienen en el problema. Se entiende este problema central, como emergente en el grupo en particular, en relación con su contexto.

- Análisis de las categorías. Guía para elaborar problemas. ¿Qué podemos aprender por un método problémico y qué no? Hacer 
un análisis de los problemas desde los núcleos de tensión que se ven implicados.

- Mentalización previa tanto por el maestro como por el estudiante, de la nueva forma de actuar. Aceptación de la incertidumbre, aceptación de la indefinición inicial de la situación. El maestro está liberado del control total de la clase, y deberá aprender a no restringir y a aceptar las diferencias.

- Diseño adecuado de los problemas con el fin de crear un estado de duda, de vacío conceptual que lleve al estudiante a preguntarse.

- El tipo de organización que se dé en las clases, se comprenderá como un orientador de las relaciones.

- La relación individuo-grupo se comprenderá como un campo de tensión potenciador del desarrollo.

- Existirá un ambiente dialógico desde el cual cada estudiante escuche, sea escuchado, experimente y observe de manera individual y colectiva, al interior del grupo.

- Se planteará una dinámica divergente-convergente, en la que cada estudiante se enfrenta al problema, explorando posibilidades diferentes, las cual socializará luego en pequeños grupos y finalmente, se llegará a un consenso en la respuesta al problema. Es posible que la convergencia no sea absoluta.

Los numerales pretenden ser generalizaciones que exigen su adecuación en contexto.

\section{Conclusiones}

Conclusiones es un decir, porque son quizá la punta de un iceberg de incertidumbres, que van quedando como moralejas, mejor, del camino vivido. Las siguientes conclusiones surgen (emergen) de la puesta en práctica de la metódica planteada:

- La inter-estructuración, más que un método, es un enfoque epistemológico de la enseñanza, que deriva, por supuesto en una metódica (conjunto de métodos, estrategias y/o principios que guardan relación entre sí, desde una concepción de la enseñanza, del aprendizaje, del conocimiento) que reconoce una dialéctica entre procesos hetero y auto-estructurantes, presentes en la dialógica de la enseñanza-aprendizaje, teniendo en común procesos de interdependen- cia, corresponsabilidad, problemicidad, que en su dialógica devela una dinámica de conflictos. El conflicto surge de la problematización que se desarrolle sobre la contradicción. Lo que hace el planteamiento problémico de la situación de enseñanza, es hacer explícita, visible la contradicción, que le lleva a crear en sí o recrear en el medio, condiciones para un nuevo equilibrio. El estudiante no se hace necesariamente consciente de la contradicción y no se desequilibra, lo cual nos reitera que la contradicción sólo se supera en la dinámica de la acción: acción de la cultura-acción del estudiante.

- La metodología problémica se ha fundamentado desde las diferentes teorías del aprendizaje, cada cual dándole un matiz diferente, un carácter distinto, y desde su perspectiva, prescribe y lee diferentes procesos en la metodología problémica. Pero es común a todas ellas la intención de reconstruir los diferentes procesos científicos retomando así la metodología de formación en el pensamiento científico. Subyacen allí, pensamos, diferentes concepciones de lo que sería hacer ciencia y del científico. Desde la educación física, un carácter problémico de la enseñanza, no apuntaría a "la formación de un científico", sino al desarrollo de una mentalidad reflexiva sobre el cuerpo, sobre las condiciones y posibilidades personales, sobre la relación con el otro, pero además, no desde la teoría (aunque también) sino desde la experiencia.

- Partimos de que una situación problémica, se genera a partir de unas "fuentes de problematización". Estas se entienden como los tejidos situacionales que en la clase son propicios para hacer explícitos unos núcleos de tensión propios del desarrollo. En el caso de la educación física, consideramos aquí diferentes fuentes de problematización: el "movimiento" en sí, la "interacción" con el compañero(s), la "comprensión" de las situaciones de juego o de instrucciones, y la "actitud personal" frente a las situaciones planteadas en clase.

- El problema del acerbo motor inicial como condición básica para la resolución de un problema motor, nos lleva a:

a. Generar referentes iniciales que permitan la asimilación de condiciones acordes con la exi- 
gencia de la situación problémica a plantear. Se ve como necesario trabajar el desarrollo funcional (capacidades motrices, patrones motores fundamentales) del movimiento, como acerbo inicial para los aprendizajes.

b. Adecuar la situación problema en la proximidad de las condiciones iniciales del estudiante. Esto es: plantear una posibilidad 1, posibilidad 2 , posibilidad 3, etc.

- Evidentemente, en la clase se observa que los estudiantes, en muchos casos tienen las condiciones necesarias para ejecutar las destrezas, pero no tienen nociones de procesos, no tienen pautas de organización, aunados a expresiones de desconfianza en sí mismos y en los compañeros, lo cual dificulta aún más la práctica corporal planteada. Esto lleva a la necesidad de plantear las condiciones iniciales para la resolución del problema. Es pertinente iniciar por la construcción de las condiciones de posibilidad, que propicien "la rotura del cascarón". Esto es: privilegiar en un primer momento las relaciones sociales que permiten el trabajo en equipo, el respeto, la puesta en práctica de un valor, lo cual hace que la tarea pierda importancia como resultado a aprender.

- Este aspecto, la problematización de la interacción con el otro, o el otro como generador de alternativas o complicaciones, debe ser un factor central en el trabajo de la clase, por cuanto el vector de la socialización es un factor clave en el desarrollo del estudiante. Pasar del movimiento mecánico, repetitivo, al hecho no tan simple de sentirlo o pensarlo, lo convierte en fuente potencial de problematización. El hecho de hacer un trote de calentamiento, pero cumpliendo la tarea de preguntarle al compañero algunos detalles de su vida, y retener los datos hasta casi el final de la clase, para socializarlos, lleva a reorientar las relaciones entre los estudiantes en un sentido proactivo.

- Cuestionar el propio saber del estudiante: ¿cuántos sentidos tenemos?, por ejemplo, genera una ruptura con su propia versión de sí mismo. Y a esa primera ruptura, seguirán otras: “y... si cada sentido busca su propia forma de placer: el oído, la música; el tacto, las texturas suaves, el olfato, los perfumes... en fin, de esos sentidos que no conocemos, ¿cuáles serán sus formas de placer? ¿Tendrá que ver algo con ellos, la práctica de los deportes extremos? ¿La búsqueda del vértigo?”. El cuestionamiento cada vez más lejos, coloca la respuesta a más distancia de la zona de seguridad. Pero el estudiante que ya ha interiorizado la pregunta, pide cuerda.

- Un valor identificado en el planteamiento de situaciones problémicas, esporádicas, pero reincidentes, es el hecho de explicitar las tensiones presentes en la clase. El problematizar un "no puedo", ponerlo en evidencia frente a las condiciones de posibilidad del propio acerbo motor, el explicitar la desconfianza existente en un compañero, exige un tratamiento dialogado, de comprensión de lo humano, pero sobre la base de lo que se espera de el, y del reconocimiento de la posibilidad de cambio. Ese hecho de hacer explicito lo oculto, permite trabajarlo más allá de la clase, desde las situaciones trabajadas en la clase.

- El reconocimiento del lenguaje, como forma de expresión directa, que posibilita el diálogo, pero más allá del carácter discursivo, en la confrontación con la práctica concreta del trabajo de clase, es un aspecto que se rescata del planteamiento de situaciones problémicas en la clase de Educación Física. El uso de otros lenguajes, gestual, metafórico, kinésico, incide en la construcción del tejido simbólico de la clase. ¿Qué le quedó de hoy para su vida?

- El hecho de que el estudiante, el grupo pueda re-direccionar el curso de la clase en un momento dado, en procesos de divergencia con lo planteado, posibilita la emergencia de procesos de co-implicación (sentirse parte activa de la clase), co-determinación (estar más abiertos a las influencias de los otros y de la tarea en sí, en tanto que pone en juego su propia influencia), y co-construcción (en un proceso donde dialécticamente se pone en juego el desarrollo, en el desarrollo de la tarea).

- El reconocimiento de la interdependencia: en la cotidianidad los estudiantes se mueven entre dos polos: dependen de sus padres, dependen de sus compañeros para soltarse luego y poder 
solos, en la invertida como tarea de una clase, por ejemplo. Pero no se atreven a aceptar esas dependencias, y persisten solos, algunos pueden, la mayoría desiste. Reconocer que se necesita del otro, es un fundamento clave del valorarlo. Pero no una dependencia pasiva, sino la interdependencia, en el aporte común. En un partido de baloncesto en la clase, al muchacho hábil le cuesta reconocer el valor del defensa, que puede que juegue menos, pero resta goles al adversario. El cuestionamiento, el hacerlo explícito, poner al defensa a subir también, lo llevan a cuestionarse y adoptar otra actitud.

\section{Referencias}

Aebli, H. (2001). Doce formas básicas de enseñar, Madrid, Narcea Editores.

Freire, P. (1983). Pedagogía del oprimido, Bogotá, Siglo XXI.

Majmutov (1984). La enseñanza problémica, La Habana, Pueblo Nuevo.

Molano, M. (1991). El juego como una estrategia pedagógica para el desarrollo integral del niño, Tesis de Grado, Facultad de Educación Física, Universidad Pedagógica Nacional.

Mosston, M. (1982). La Enseñanza de la Educación Física, Barcelona, Paidos.

Morin, E. (1992). El conocimiento del conocimiento, Madrid, Ediciones Cátedra.

Parlebas, P. (2001). Juegos, deportes y sociedades. Diccionario de Praxiología motriz, Barcelona, Paidotribo.

Piaget, J. (1998). Seis estudios de psicología, Bogotá, Panamericana.

Restrepo, L. (1993). 'El problema de la socialización y la cultura'. En Urdimbres y Tramas Culturales, Bogotá, Corprodic.

Tudge, J., En Moll, L. (1993). Vygotsky y la educación, Buenos Aires, AIQUE.

Zubiría Samper, M. (2005). Enfoques Pedagógicos y Didácticas contemporáneas, Bogotá, FIDC.

\section{Gráficos:}

Diapositivas construidas en Power Point, al interior del grupo de investigación Complexus. Didáctica de la Educación Física y Desarrollo Humano. 